\title{
Modelling multivariate disease rates with a latent structure mixture model
}

\author{
P.J.Hewson ${ }^{* \dagger}$ and T.C. Bailey ${ }^{\ddagger}$
}

October 3, 2008

\begin{abstract}
There has been considerable recent interest in multivariate modelling of the geographical distribution of morbidity or mortality rates for potentially related diseases. The motivations for this include: investigation of similarities or dissimilarities in the risk distribution for the different diseases, as well as 'borrowing strength' across disease rates to shrink the uncertainty in geographical risk assessment for any particular disease. A number of approaches to such multivariate modelling have been suggested and this paper proposes an extension to these which may provide a richer range of dependency structures than those encompassed so far. We develop a model which incorporates a discrete mixture of latent structures and argue that this provides potential to represent an enhanced range of correlation structures between diseases at the same time as implicitly allowing for less restrictive spatial correlation structures between geographical units. We compare and contrast our approach to other commonly used multivariate disease models and demonstrate comparative results using data taken from cancer registries on four carcinomas in some 300 geographical units in England, Scotland and Wales.
\end{abstract}

Keywords: Latent Structure; Mixture Model; Multivariate Disease Rates; Factor Analysis.

\section{Introduction}

The literature in spatial epidemiology contains a growing number of references to multivariate modelling of the geographical distribution of morbidity or mortality rates for potentially related diseases. Dabney and Wakefield (2005) suggest that the two main motivations for this interest are firstly, to explore similarities or dissimilarities in the geographical risk distribution for the different diseases, and, secondly, to 'borrow strength' across disease rates to shrink the uncertainty in geographical risk assessment for any one of the individual diseases. Regardless of the relative balance of interest between these two motivations, it is clear that accurate inference in respect of either of them will be limited if the structure of the multivariate model used is inadequate and/or its related exchangeability assumptions are unrealistic. The possible dependency structure, either between diseases, or across geographical units, should not be overly

* School of Mathematics and Statistics, University of Plymouth, Drake Circus, Plymouth PL4 8AA tel: +44 (0)1752 586870, fax: +44 (0)1752 232780, email: paul.hewson@plymouth.ac.uk

${ }^{\dagger}$ Corresponding Author

${ }^{\ddagger}$ School of Engineering, Computing and Mathematics, University of Exeter, North Park Road, Exeter EX4 4QE tel: +44 (0)1392 725223, fax: +44 (0)1392 217965, email: t.c.bailey@exeter.ac.uk 
restricted or simplified by the model. For example, it may not be the case that relationships between diseases are the same in, say, rural versus urban environments, nor that dependency between disease rates in neighbouring small areas will be homogeneous at a larger geographical scale where spatial discontinuities may well be present. The model structure should provide a sufficiently rich range of dependency structures to encompass such possibilities.

Various approaches to spatial modelling of multivariate disease rates have been proposed. Broadly these may all be characterised as generalised linear mixed models (GLMMs) of varying descriptions in which the dependence structure not explained by covariates is represented in terms of random effects which are correlated between diseases and across geographical units.

The Multivariate Conditional Autoregressive (MCAR) model is one popular approach to consider in this context and Gelfand and Vounatsou (2003) discuss MCAR formulations suitable for dealing with multivariate disease rates in small areas. More recently, further results for these kinds of models are given by Song et al. (2006) who were concerned with multivariate area counts of road injuries. We discuss MCAR models further in Section 2 as a basis for providing comparative results from the alternative models we propose, but it is worth noting here that MCAR models typically employ a Wishart prior when modelling the correlation in random effects and there are some concerns with the use of this prior. For example Yang and Berger (1994) note that having a single parameter for the degrees of freedom implies that prior knowledge is the same for the relationships between all variables. There are suggestions in the literature for alternative priors (e.g. Leonard and Hsu, 1992; Daniels and Kass, 2001), but MCAR formulations remain difficult to fine tune because the correlations in random effects between diseases and/or across spatial units are not easy to disentangle.

For that reason (and others, such as ease of extensibility) models which incorporate more explicit latent structure also feature in multivariate modelling of disease rates. Held et al. (2005) review a range of approaches to joint disease modelling and examine shared latent processes in some detail. An early example of such a model, considering Minnesota cancer rates, was reported by Wang and Wall (2003) who identified a shared spatial process related to three of the four cancer rates they investigated. Their model used a single latent variable following a Gaussian Conditional Auto-Regressive (CAR) distribution. A less flexible model had been proposed earlier by Knorr-Held and Best (2001), which was constructed to accord with their primary interest of identifying a shared spatial component in the geographical distribution of bivariate disease rates. More recently, Liu et al. (2005) have proposed a Structural Equation Model (SEM) for cancer rates where the three cancers have a single shared spatially structured latent variable. The aim of that model was to relate the shared risk to a number of disease determinants, but again one principal goal was to model diseases having a common spatial component. Latent structure models are not restricted to area applications. Christensen and Amemiya (2002) have suggested an approach applicable to point data which has been illustrated by Minozzo and Fruttini (2004) who examined bivariate point measures of types of diabetes morbidity. Similar models are also seeing use outside cancer epidemiology. For example, Christensen and Amemiya (2003) demonstrate an application in agriculture and Hogan and Tchernis (2004) report an application considering variation in socio-economic conditions. Further proposals include modelling longitudinal dependencies in the latent variables (Dunson, 2003) as well as data of mixed response types (Dunson and Herring, 2005).

MCAR versus explicit latent structure aside, there are two assumptions which dominate most of these previously described multivariate models. First, that spatial dependency across small areas is essentially "smooth" and not subject to global spatial discontinuities. Second, that it is reasonable to assume that a single relationship between diseases counts applies to all 
types of areas. In some (perhaps many) applications the dependence structure may well be more complex than that implied by these rather broad assumptions. Normand et al. (1997) highlight that in the absence of adequate covariate information, simple exchangeability assumptions across areas may not be valid in many of the GLMMs used to analyse healthcare provision. In a multivariate setting these exchangeability concerns across areas remain, but are compounded by additional concerns over whether the dependence structure between diseases varies geographically. For example, multivariate models seeking to reduce uncertainty by borrowing strength across variables and which assume that the same dependence structure between diseases applies to all areas, may result in unreasonably precise predictions about the uncertainty associated with relative risks.

We therefore propose models for use in such contexts which provide a richer range of dependency structures than encompassed by previous approaches. Our models are based on latent structure mixtures and we argue that incorporating a discrete mixture into the latent structure loadings in the model simultaneously provides potential to represent an enhanced range of correlation structures between diseases, at the same time as allowing for less restrictive spatial correlation structures between geographical units. Proposals have already been made to utilise mixtures in joint disease modelling, such as the Knorr-Held and Best (2001) bivariate model. However, we will consider a more general approach applicable where there are more than two observed variables. The structure we propose could be considered similar to a 'mixture of factor analysers'. Such models (mostly for Gaussian responses) have been reported in other contexts in the statistical literature and elsewhere. For example, McLachlan and Peel (2000) discuss mixtures of factor analysers, Lee and Song (2003) report on mixtures in relation to Structural Equation Modelling, and Viroli (2004) describes 'independent factor analysis' based upon approaches developed in the signal processing literature which use mixtures to model non-Gaussian latent variables.

The outline of the remainder of this paper is as follows. We will develop our models and describe fitting strategy in Section 2. In Section 3 we introduce an illustrative data set on which to compare results and which concerns four cancers in some 300 geographical units in England, Scotland and Wales. We present comparative model results for these data in Section 4 and then go on to discuss the implications and our conclusions in Section 5.

\section{Models}

In modelling multivariate count data, such as recorded cases of a number of diseases observed on the same spatial units (e.g. areas), it is typical, as mentioned in Section 1, to represent the dependence structure not explained by covariates in terms of correlated random effects. We suggest that it is preferable to formulate these correlations in terms of an explicit latent structure which is not dissimilar to the kinds of latent structure arising in factor analysis. Many insights into the properties of GLMMs for multivariate disease rates can be gained from studying recent developments in factor analysis. For example, count data factor analysis was proposed nearly a decade ago by Ogasawara (1996) and formalised in an econometric framework. More recently Wedel et al. (2003) extended the range of latent structure distributions and gave results on the role of the common and unique components. Whilst identifiability issues have caused some concern, a number of recent contributions from the graphical modelling literature (Grzebyk et al., 2004) assist in terms of modelling correlated common or unique elements. It should also be noted that factor analysis has been enjoying somewhat of a methodological renaissance in a Bayesian setting (Aitkin and Aitkin, 2005) and a number of useful results 
have emerged. For example Aguilar and West (2000) indicate that having too many latent variables for Gaussian data is not a problem if the goal of a model is to make good predictions, whereas many problems in under-fitting can be anticipated if too few latent variables are used. The development of our latent structure mixture model for joint disease modelling in this section is encouraged by these results and draws upon our belief in the value of viewing correlated random effects in a factor analysis framework.

The basic structure of the problem we consider is that we have data, $y_{i j}$, representing the number of cases in area $i$ for disease $j(i=1, \ldots, n ; j=1, \ldots, p)$. The corresponding expected number of cases $e_{i j}$ is also known, this being based on age/sex standardised rates for the whole of the study region, or for some appropriate alternative reference population (equivalently, we may know $y_{i j}$ along with the standard morbidity ratio (SMR) $\frac{y_{i j}}{e_{i j}}$, for disease $j$ in area $i$ ). Where appropriate we will refer to the vector of disease counts in each area as $\boldsymbol{y}_{i}=\left(y_{i 1}, \ldots, y_{i p}\right)$ and the corresponding vector of expected counts as $\boldsymbol{e}_{i}=\left(e_{i 1}, \ldots, e_{i p}\right)$. In a practical setting we may well also have a vector of covariates $\boldsymbol{x}_{i}(i=1, \ldots, n)$ measured in each area, but for simplicity of exposition we will assume throughout this paper that such covariates are not available. If required these can be included into the models we develop in an obvious and straightforward fashion.

It is usual to assume the disease counts follow a Poisson distribution so that:

$$
p\left(y_{i j} \mid \lambda_{i j}\right) \sim \operatorname{Pois}\left(e_{i j} \lambda_{i j}\right)
$$

with the mean vector, $\boldsymbol{\lambda}_{i}=\lambda_{i 1}, \ldots, \lambda_{i p}$, in each area then being modelled through an appropriate link function by a suitable linear predictor. In developing our modelling framework we build upon proposals made by Wang and Wall (2003) mentioned in Section 1 which use a simple linear predictor involving a single area specific latent variable with a disease specific loading, so that:

$$
\log \left(\lambda_{i j}\right)=\phi_{i} \delta_{j}
$$

where $\delta_{j}$ is the disease specific loading and $\phi_{i}$ is the area specific latent (unmeasured) variable. The latent variable is assumed to follow a Conditional Auto-Regressive Gaussian (CAR) distribution over the areas, so that: $\phi_{i} \mid \phi_{j \neq i} \sim \mathrm{N}\left(\sum_{j \neq i} w_{i j} \phi_{j}, \sigma^{2}\right)$ where $\left\{w_{i j}\right\}$ are elements of an $n \times n$ adjacency matrix with row sums standardised to unity in which $w_{i j} \neq 0$ denotes that areas $i$ and $j$ are neighbours. The associated joint density for $\left(\phi_{1}, \ldots, \phi_{n}\right)$ is then improper, but the full conditionals are proper, and we prefer this 'intrinsic' version of the CAR because the mean of $\phi_{i}$ is then precisely the mean of its neighbours. Alternative proper CARs (such as that used by Wang and Wall, 2003) include an additional spatial association term in the formulation, but this then yields a conditional mean for each area which is proportional rather than equal to the average of its neighbours. Instead of that, we handle our preferred improper intrinsic CAR through the conventional approach of actually fitting $\phi_{i}=\alpha+\phi_{i}^{\star}$, where $\alpha$ has a flat distribution over the whole real line. Note further that we have no 'islands' (areas that have no neighbours ) in our analyses, otherwise further modifications to the CAR formulation may be necessary.

It is important to appreciate that model (1) can reflect implicit correlation between diseases within an area (through the shared latent variable) and also spatial correlation across areas (through the CAR). However, the simple structure only provides for a limited range of correlation structures between diseases (same for all areas) and makes possibly unrealistic exchangeability assumptions about the spatial dependence (no global spatial discontinuity). One or both of these criticisms can also be applied to proposed variations on this simple latent variable modelling structure, such as those referenced in Section 1 . We therefore consider 
ways to extend this structure to provide more complex possibilities for dependencies between diseases and across areas.

First, to allow potential for a more complex dependence structure between diseases, we include $q$ latent variables. So that the model becomes:

$$
\log \left(\lambda_{i j}\right)=\sum_{h=1}^{q} \phi_{i h} \delta_{h j}
$$

where $\delta_{h j}$ is a disease specific loading for area specific latent variable $\phi_{i h}(h=1, \ldots, q)$. We can express this more succinctly as:

$$
\log \left(\boldsymbol{\lambda}_{i}\right)=\phi_{i} \boldsymbol{\Delta}
$$

where it is understood that $\log \left(\boldsymbol{\lambda}_{i}\right)=\left(\log \lambda_{i 1}, \ldots, \log \lambda_{i p}\right)$ and where $\phi_{i}=\left(\phi_{i 1}, \ldots, \phi_{i q}\right)$ is the vector of latent variables for area $i$ and $\Delta$ is the $q \times p$ matrix of loadings:

$$
\boldsymbol{\Delta}=\left(\begin{array}{rrr}
\delta_{11} & \ldots & \delta_{p 1} \\
\vdots & \ddots & \vdots \\
\delta_{1 q} & \ldots & \delta_{p q}
\end{array}\right)
$$

Expanding the number of parameters in this way raises identifiability problems, we choose to follow Lopes and West (2004) and constrain the loading matrix $\Delta$ so that $\delta_{h j}=0$ if $j>h$ and $\delta_{j j}>0$ for $j=1, \ldots, p$ in order to remove the rotational indeterminacy.

Second, to incorporate a richer range of dependency across areas we further extend model (2) to a mixture model across $s$ sets of $q$ latent variables in each area, with $\phi_{i h}^{(k)}(h=1, \ldots, q)$ denoting the $k t h$ set of latent variables. So that the model becomes:

$$
p\left(y_{i j} \mid \lambda_{i j}^{(1)}, \ldots, \lambda_{i j}^{(s)}\right) \sim \sum_{k=1}^{s} \pi_{k} \operatorname{Pois}\left(e_{i j} \lambda_{i j}^{(k)}\right)
$$

with $\pi_{k}$ denoting mixing probabilities $\left(\sum_{k} \pi_{k}=1\right)$ and with:

$$
\log \left(\boldsymbol{\lambda}_{i}^{(k)}\right)=\phi_{i}^{(k)} \boldsymbol{\Delta}^{(k)}
$$

where $\boldsymbol{\Delta}^{(k)}$ is a $q \times p$ matrix of loadings for the the $k t h$ set of latent variables $(k=1, \ldots, s)$ and each such matrix is subject to the constraints described earlier. The latent variables $\phi_{i h}^{(k)}$ are assumed to follow independent $\mathrm{N}\left(\mu_{h}^{(k)}, 1\right)$ distributions for $(k=1, \ldots, s),(h=1, \ldots, q)$ and $(i=1, \ldots, n)$. So each area is now a mixture of $s$ types of areas, with each type of area being associated with a different set of $q$ latent variables and corresponding loadings.

There is no explicit spatial dependence in the above formulation, but implicit spatial dependence arises through groups of areas being free to share a similar pattern of mixing probabilities over the sets of latent variables and loadings. This type of spatial dependence is potentially very flexible since it does not necessarily impose undue global spatial smoothness. However, as an alternative formulation we can also consider introducing a degree of explicit spatial dependence by retaining a mixture of loadings, whilst replacing the mixture of latent variables with a single set $\phi_{i h}(h=1, \ldots, q)$, so that:

$$
\log \left(\boldsymbol{\lambda}_{i}^{(k)}\right)=\phi_{i} \Delta^{(k)}
$$


We then take each latent variable $\phi_{i h}(h=1, \ldots, q)$ to follow an intrinsic CAR distribution over the areas $i$. We continue to regard the $q$ latent variables within an area as independent, which is both consistent with the assumptions in factor analysis and also avoids potential identifiability problems with respect to the spatial structuring.

Finally, we note that it is necessary to incorporate additional unstructured area and disease specific random effects into the linear predictor of both our formulations above in order to deal with possible overdispersion. These additional latent variables are effectively equivalent to 'uniqueness' in the traditional factor analysis literature.

So the mixture model for both both latent variables and loadings then becomes:

$$
\log \left(\boldsymbol{\lambda}_{i}^{(k)}\right)=\phi_{i}^{(k)} \boldsymbol{\Delta}^{(k)}+\boldsymbol{\zeta}_{i}
$$

where the random effects, $\boldsymbol{\zeta}_{i}=\left(\zeta_{i 1}, \ldots, \zeta_{i p}\right)$ are independent zero mean Gaussian with variances drawn from inverse Gamma(0.1,0.1) hyperpriors.

While the equivalent for the mixture model for loadings only is:

$$
\log \left(\boldsymbol{\lambda}_{i}^{(k)}\right)=\phi_{i} \boldsymbol{\Delta}^{(k)}+\boldsymbol{\zeta}_{i}
$$

with the same distribution as above for $\boldsymbol{\zeta}_{i}$.

Models (3) and (4) involve both unknown numbers of latent variables and mixture components. We adopt a fitting approach based on MCMC, but there is considerable complexity in using dimension changing methods (e.g. RJMCMC) even with just unknown numbers of latent variables (e.g. Lopes and West, 2004) let alone when this is compounded with an unknown number of mixture components. We therefore follow suggestions made by Green (2003) and use a strategy whereby distinct models are fitted to distinct dimensionalities. In selecting the number of mixture components we draw on similarities between our latent structure mixture and the 'mixture of factor analysers' model (McLachlan and Peel, 2000, Chap 8). We note that in the machine learning literature, 'Variational Bayes' approaches are used to fit 'mixtures of factor analysers' (Ghahramani and Beal, 2000; Beal and Ghahramani, 2003) which are essentially equivalent to minimising the Kullback Leibler distance between the factorised approximation and the joint posterior. We have therefore selected a strategy based on the Kullback-Leibler distance in order to select the number of mixture components. We use this measure to assess the distance between our fitted model and a model which assumes the two closest mixture components have been merged. An approximation to the Kullback-Liebler distance can be generated as a byproduct of Gibbs sampling (Sahu and Cheng, 2003) and we use this to guide model selection with respect to the number of substantive mixture components which may be supported by the data.

Using mixture models as in both the above formulations, raises a number of fitting and identifiability issues (McLachlan and Peel, 2000). We follow Celeux et al. (2000) by not placing any constraints on the ordering of the mixture group means and deal with label-switching by postprocessing the output of our MCMC sampler. Mixture group memberships for loadings and latent variables (where applicable) were assumed to be categorical variables with a Dirichlet prior for $\pi_{k}$, the probability of belonging to a particular group, given by:

$$
p\left(\pi_{1}, \ldots, \pi_{s}\right)=\frac{\Gamma\left(\alpha_{1} \ldots \alpha_{s}\right)}{\Gamma\left(\alpha_{1}\right) \ldots \Gamma\left(\alpha_{s}\right)} \pi_{1}^{\alpha_{1}-1} \ldots \pi_{s}^{\alpha_{s}-1},
$$

where $\alpha_{j}$ represents the prior group weights for each of the $k=1, \ldots, s$ mixing components. Each element $\delta_{i j}^{(k)}$ of each of the loading matrices $\boldsymbol{\Delta}^{(k)}$ is independently drawn from a normal prior with zero mean and with its own variance, but with the latter having a restrictive 
Inverse Gamma(1,1) hyperprior. Eberly and Carlin (2000) note that individual additive random effects within a linear predictor may not be identifiable even though the sum of these latent variables can be identified. Visual checks were therefore made for convergence of identifiable nodes within the model, supported by more formal convergence checking using Gelman and Rubin's R (Gelman and Rubin, 1992), Geweke's statistics (Geweke, 1992) and Heidelberger and Welch's (Heidelberger and Welch, 1983) statistic. These are slow models to fit, running multiple chains is therefore somewhat time consuming but essential given the level of cross-correlation possible due the model formulation.

Overall model fit was assessed by a number of measures. In addition to assessing mixture group membership in terms of Kullback-Leibler distance, we further used visual inspection of the Pearson residuals $\frac{y_{i j}-\left(e_{i j} \hat{\lambda}_{i j}\right)}{\sqrt{e_{i j} \hat{\lambda}_{i j}}}$ where $\hat{\lambda}_{i j}$ denotes the posterior mean estimate of $\lambda_{i j}$. We used Posterior Predictive Loss as proposed by Gelfand and Ghosh (1998) as well as a variant on this proposed by Gneiting and Raftery (2007) which enjoys the advantage of being a Proper scoring rule.

Noting that the Posterior Predictive Loss can be estimated from the MCMC output and requires the addition of a forward sampling step where the data are replicated by sampling without replacement, denoted here as $y_{r e p}$. Posterior Predictive Loss is then based on departure of the replicated data from the observed data, $y_{o b s}$, penalised according to the variance in the replicates. Throughout this paper we follow Banerjee et al. (2004) who assume a squared loss function and give Posterior Predictive Loss (PPL) for penalising weight, $\kappa$, as:

$$
D_{\kappa}=\frac{\kappa}{\kappa+1} \sum\left(\mathrm{E}\left[y_{r e p} \mid y_{o b s}\right]-y_{o b s}\right)^{2}+\sum \operatorname{Var}\left[y_{r e p} \mid y_{o b s}\right]
$$

In essence the first component of $D_{\kappa}$ (denoted $G$ in subsequent sections of this paper) is a goodness of fit component, whereas the second component (denoted $P$ in subsequent sections) is a penalty term (as the model is over-fitted the variance in the replicates will tend to inflate due to multicollinearity).

The modification to this measure suggested by Gneiting and Raftery (2007) is also based only on first and second moments but is proper. This measure, examined specifically in respect of count data by Czado et al. (2007) is given by:

$$
S=-\left(\frac{y_{o b s}-E\left[y_{r e p} \mid y_{o b s}\right]}{\sqrt{\operatorname{Var}\left[y_{\text {rep }} \mid y_{o b s}\right]}}\right)^{2}-\log \operatorname{Var}\left[y_{r e p} \mid y_{o b s}\right]
$$

We refer to this proper score as the "Dawid and Sebastiani score" following earlier work reported in Dawid and Sebastiani (1999).

To assess comparative performance of the latent structure mixture models proposed in this section, we will contrast results in subsequent sections with those from a standard Multivariate Intrinsic CAR (MCAR) with an additional random effect allowing for over-dispersion. As mentioned in Section 1, such models are very commonly used for joint disease modelling. In its simplest form that model may be expressed as:

$$
\log \left(\boldsymbol{\lambda}_{i}\right)=\boldsymbol{\nu}_{i}
$$

where the vector of random effects $\boldsymbol{\nu}_{i}=\left(\nu_{i 1}, \ldots, \nu_{i p}\right)$ has a Multivariate CAR over the areas, so that $\boldsymbol{\nu}_{i} \mid \boldsymbol{\nu}_{j \neq i} \sim \operatorname{MVN}\left(\sum_{j \neq i} w_{i j} \boldsymbol{\nu}_{j}, \boldsymbol{\Sigma}_{\mu}\right)$, with adjacency weights, $\left\{w_{i j}\right\}$, as before, and with 
$\boldsymbol{\Sigma}_{\mu}$ being a $p \times p$ covariance matrix between diseases. As earlier we use this improper intrinsic form of the MCAR distribution and handle this by actually fitting $\boldsymbol{\nu}_{i}=\boldsymbol{\alpha}+\boldsymbol{\nu}_{i}^{\star}$, although it should be noted that there have been recent developments in the literature in terms of defining proper multivariate CARs (e.g. Gelfand and Vounatsou, 2003; Song et al., 2006). In order to allow for spatially unstructured overdispersion as well as the spatially structured overdispersion modelled by this multivariate CAR we incorporate an additional unstructured multivariate Normal distribution to account for any over-dispersion not already dealt with. This comparison model therefore has the following linear predictor:

$$
\log \left(\boldsymbol{\lambda}_{i}\right)=\boldsymbol{\nu}_{i}+\boldsymbol{\zeta}_{i}
$$

where in addition to the terms indicated in formula 6 we now have $\boldsymbol{\zeta}_{i} \sim M V N\left(\mathbf{0}, \boldsymbol{\Sigma}_{\zeta}\right)$, where $\Sigma_{\zeta}$ is assumed to follow an inverse Wishart distribution with $j=6$ degrees of freedom.

These MCAR models are readily fitted using available code within the WinBUGs software package (Spiegelhalter et al., 1998). We therefore also used WinBUGs to fit both of our proposed latent structure mixtures models (i.e. models 3 and 4). Whilst this may be somewhat slower than using customised code, it does offer a benchmark which allows results from models to be compared independently of algorithmic developments. To facilitate model comparisons we focus on the posterior predictive distribution of the relative risks for each disease for each area studied. Using MCMC, such results can easily be obtained as part of model fitting and are directly comparable across the different models considered.

We validated the performance of our models by means of out of sample predictions. For each cancer site in turn, we removed one tenth of the observations at random, and fitted the model to these data. The posterior predictive density for these deleted observations was collected during the model fitting process, and compared with the observed data. Visual checks as well as the posterior predictive score (formula 5) were used to assess the out of sample predictive behaviour.

\section{Data}

As a illustrative application to compare model performance, we consider data on reported numbers of cases of four types of cancer in some 300 geographical units covering England, Wales and Scotland. These data were obtained from the 9 cancer registries in England as well as the cancer registries in Scotland and Wales and comprise the number of cases reported between 1999 and 2001 of, 'Lung cancer' (ICD-10 classified sites C33-C34, i.e. Trachea, Bronchus and Lung cancer), 'Oral cancer' (C00-C14, i.e. Lip, Oral Cavity and Pharynx), Breast cancer (C50) and Cancer of the Cervix (C53). Data were collected on prevalence for males and females for the first two cancers, but only for females in respect of breast and cervical cancer. Indirect age standardisation (Clayton and Kaldor, 1987) was used to estimate associated expected morbidity based on quinary age bands for the whole of the study region.

These data refer to the smallest administrative geographical unit available, i.e. the 303 Primary Care Trusts (PCT) in England, the 22 Local Health Boards in Wales and the 14 National Health Service Boards in Scotland. For convenience we will subsequently refer to all such units by the name given to the majority, namely 'PCTs'. The English and Welsh entities are comparable in size, for example the mean population within an English PCT is 163,000 with a minimum 63,700 and a maximum 372,600 whereas the Welsh Local Health Board mean population was 131,900 with a minimum of 56,500 and a maximum of 310,300 . Scotland is dominated by 
a couple of very large NHS Boards, the mean population was 720,320 with a minimum of 38,400 and a maximum of $1,736,300$. Some caution may therefore be needed when comparing results from England and Wales with those of Scotland due to aggregation effects alone. To avoid any difficulties in the spatial modelling, we concentrate our efforts here on those 335 'PCTs' which are entirely based on the mainland, i.e we exclude islands. It should be noted for later reference that one of the mainland PCTs in Cornwall (the far South West of our maps) contains an aggregate of data from the Isles of Scilly. We treat these Islands as a composite part of their mainland PCT.

Basic information about the number of cancers registered under each diagnosis in each PCT are contained in Table 1 . As is usual with administratively collected data, there are some provisos over accuracy. Particular problems with UK cancer registry data are documented in Best and Wakefield (1999), and we note that it may not be entirely reasonable to assume that each cancer registry collects the data in exactly the same way.

\begin{tabular}{rrrrrrr}
\hline & Oral $(\mathrm{F})$ & Lung $(\mathrm{F})$ & Oral $(\mathrm{M})$ & Lung $(\mathrm{M})$ & Breast & Cervix \\
\hline Mean & 16.25 & 132.81 & 28.65 & 205.06 & 359.99 & 26.68 \\
Standard Deviation & 11.62 & 107.06 & 23.04 & 136.67 & 184.47 & 17.62 \\
Minimum & 1 & 29 & 3 & 41 & 107 & 4 \\
Maximum & 133 & 1333 & 276 & 1653 & 1832 & 175 \\
\hline
\end{tabular}

Table 1: Summary information on the mean, standard deviation, minimum and maximum number of cancer cases registered for each of the six diagnosis groups in each of the 335 non-island PCTs in England, Scotland and Wales

Table 2 gives the observed correlation coefficients between the various cancer rates. It can be seen for example that Lung cancer rates are highly correlated between males and females (0.88), the same is not so true of oral cancer rates (0.31). The upper right panel of Figure 1 provides the same information in graphical form. The lower left panel of the pairwise scatterplots contains information on the possible group memberships identified by modelling, these results will be explained later.

\begin{tabular}{rrrrrrr}
\hline & Oral (F) & Lung (F) & Oral (M) & Lung (M) & Breast & Cervix \\
\hline Oral (F) & 1.00 & 0.23 & 0.31 & 0.24 & 0.04 & 0.22 \\
Lung (F) & 0.23 & 1.00 & 0.52 & 0.88 & -0.32 & 0.47 \\
Oral (M) & 0.31 & 0.52 & 1.00 & 0.52 & -0.18 & 0.35 \\
Lung (M) & 0.24 & 0.88 & 0.52 & 1.00 & -0.39 & 0.49 \\
Breast & 0.04 & -0.32 & -0.18 & -0.39 & 1.00 & -0.18 \\
Cervix & 0.22 & 0.47 & 0.35 & 0.49 & -0.18 & 1.00 \\
\hline
\end{tabular}

Table 2: Observed correlation between cancer rates for the four cancers, male and female data shown separately

\section{Results}

Models were fitted as described in Section 2, using the priors and convergence criteria indicated there. A standard burn in period of 50,000 iterations was used, a further 100,000 samples 


\section{Pairwise scatter plots tor SMR tor six cancer site:gender variables}

$0.5 \quad 1.5$
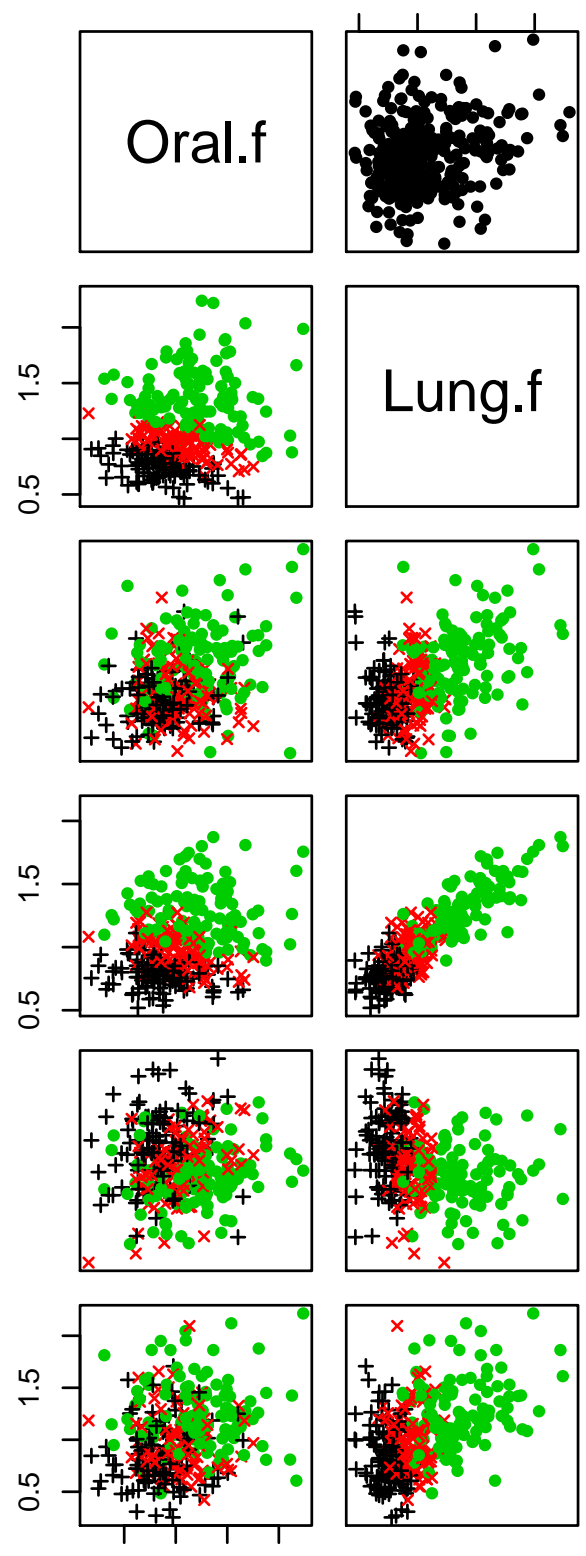

$0.5 \quad 1.5$
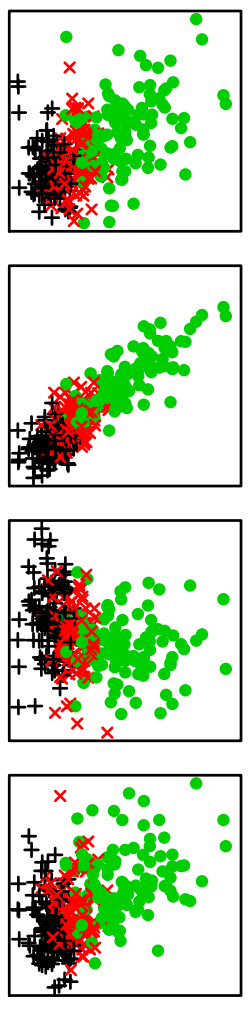

$0.5 \quad 1.0 \quad 1.5 \quad 2.0$
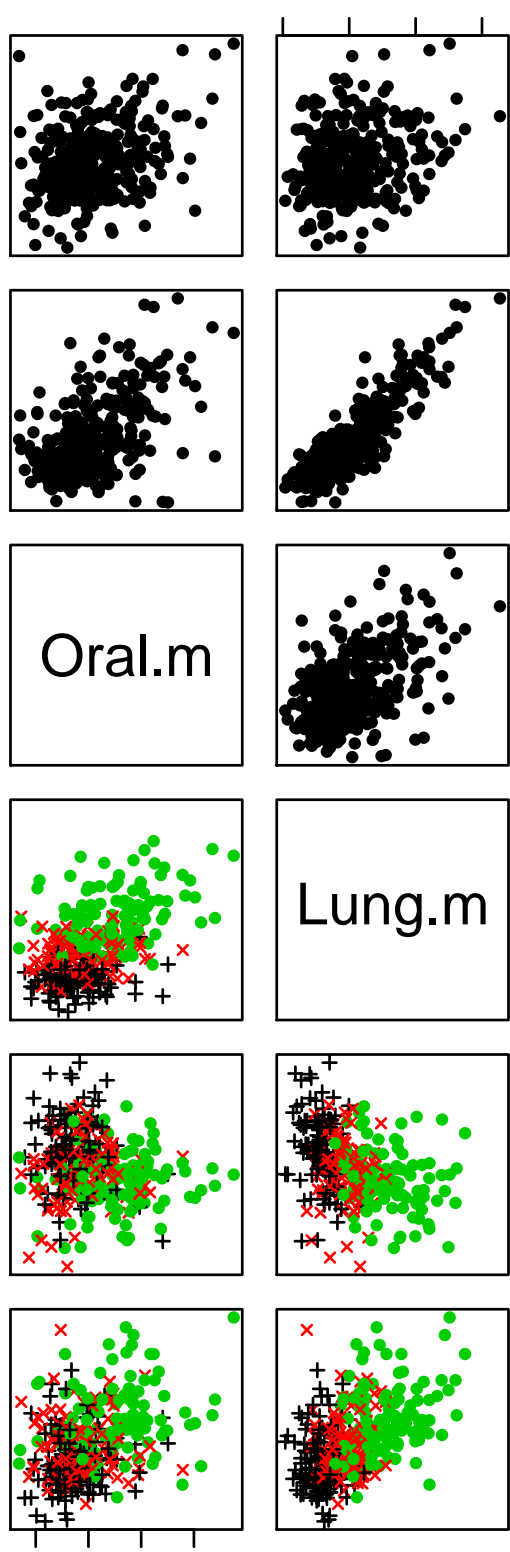
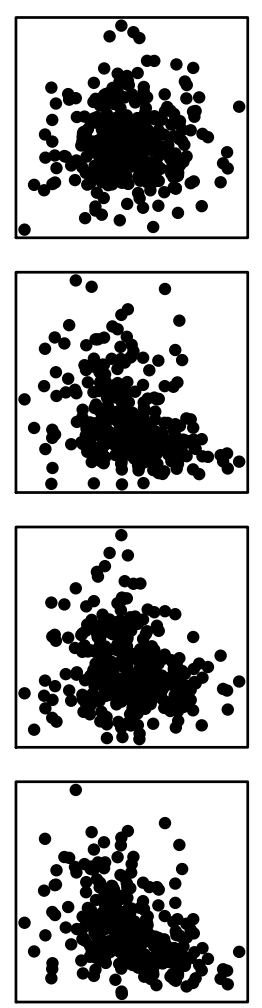

$0.5 \quad 1.5$

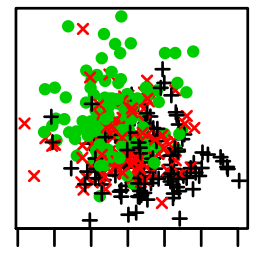

$0.7 \quad 0.9 \quad 1.1 \quad 1.3$
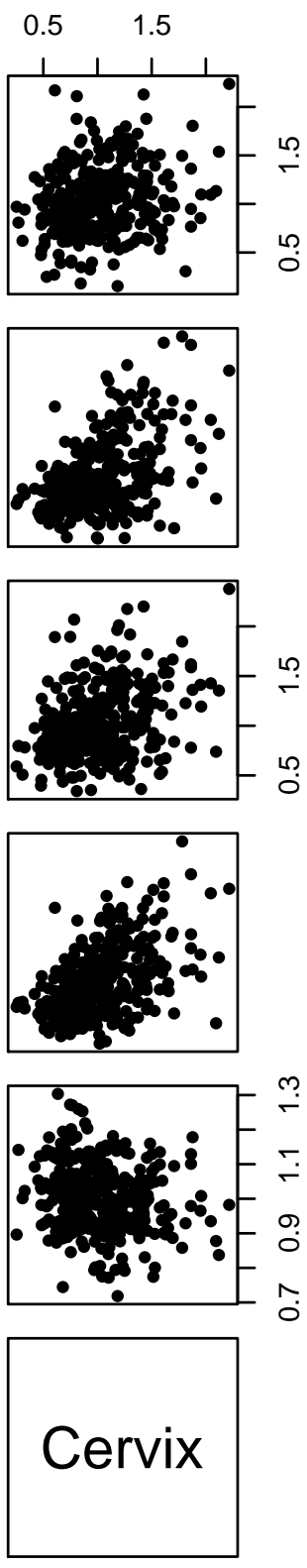

Figure 1: Pairwise scatter plots for six cancer rates based on indirect age standardisation; the plots below diagonal have been shaded to illustrate the posterior probability of belonging to one of the two groups we tentatively identify later, posterior probability $<33 \%$, between 33 and $66 \%$ and $>66 \%$ and are explained in Section 4 
thinned by a factor of 20 were used for posterior inference. As mentioned previously, it is useful (although not very fast) to compare established models with our proposals using the WinBUGs software (Spiegelhalter et al., 1998) and all results reported here were obtained in that way.

\subsection{MCAR Model}

We consider here results from the MCAR model incorporating additional non-spatially correlated random effects (i.e. model 7). The Dawid and Sebastiani (posterior predictive proper) score was $-12,107.4$ (we note that this was lower than the Dawid and Sebastiani score obtained from a model having only the MCAR structured random effects which was estimated as -15151.9) The posterior deviance for this MCAR model 7 had a mean of 13980.0 with a $95 \%$ credible interval $13880.0,14050.0$. Finally, while it is not a proper score, full results for the posterior predictive loss (PPL) are given as these do allow a decomposition into fit $(P=23,625.6)$ components which will be cross-referenced later.

It should be clearly noted that whilst we used priors that were sceptical as to the amount of shrinkage across variables, i.e. for the results presented here these inverse Wishart matrices were diagonal with elements set to 0.02 and the degrees of freedom set to six, the number of variables being modelled, these models are sensitive to prior specification of the between disease dependence structure. For example, when working with an MCAR model lacking the unstructured random effect, increasing the degrees of freedom for the Wishart prior to its maximum possible yields a fit element of $G=396,975.5$ whereas leaving the degrees of freedom equal to six but setting the diagonal elements equal to 0.0002 yields a fit element of $G=188,953.9$. This deterioration in fit is also clearly seen when using the Dawid and Sebastiani score.

\subsection{Latent Structure Mixture Spatial Model}

We now turn attention to the version of our proposed latent structure model which includes explicit spatial structure (i.e. model (4)). We fitted a range of models with differing numbers of latent variables and differing numbers of mixture components. Given $p=6$ disease counts we considered all the classically identifiable possibilities, i.e. $k=1, \ldots, 3$ latent variables. It was found feasible to fit a three latent variable model and we prefer that both because it has the greatest potential to model a complex dependence structure and because it has the lowest posterior predictive score.

The posterior predictive score decreases as the number of mixture components increases. For example, with three latent variables and three mixture groups the Dawid and Sebastiani score was $-8,923.9$ whereas with three latent variables and five mixture groups the score was reduced slightly to $-8,875.9$. Nevertheless, these all compare well with the fit from the MCAR model with overdispersion, reported earlier as $-12,107.4$.(i.e. model 7 ).

The DIC is difficult to calculate for mixture models, but the posterior deviance is essentially the same for the two models, having a mean of 13840.0 and $95 \%$ credible interval of $(13730.0,13950.0)$ for the three mixture group model and a mean of 13840.0 with a $95 \%$ credible interval of $(13730.0,13940.0)$ for the five mixture group model. This is also similar to the posterior deviance seen with the two MCAR models.

As with the MCAR models, examining the non-proper posterior predictive loss score does 
provide some further information on the model fitting behaviour. Specifically, with respect to the fit term of the posterior predictive loss $G$ decreases as both the number of latent variables increases and as the number of mixture terms increases. For example, for three latent variables and three mixture components the fit component of the PPL was $G=59,608.4$ whereas with five mixture components it was $G=56,079.3$. The apparently improved fit of the latter comes with the cost of a very modest increase in the penalty term $P$ from $24,493.1$ in the three mixture component (three latent variable) model to $24,589.2$ in the five mixture component (three latent variable) model. In both cases, the fit terms $G$ are considerably lower than the value estimated for the benchmark MCAR model which has $G=152,931.4$ although the estimate of the corresponding penalty term for the MCAR model was slightly lower at $P=22,996.4$.

We also fitted different numbers of mixture components in a systematic manner, examining the approximate Kullback-Leibler distance between each model and a collapsed model with the two closest mixture components merged. This provided no evidence for the inclusion of any mixture components. Hence, whilst we observe that the model fit improved with increasing numbers of mixture components (at the expense of a modest increase in the penalty term), this appears to be essentially 'non-parametric' - the mixture components overlap and do not necessarily have any substantive meaning.

For further examination, we select the three mixture, three latent variable model for reasons for relative parsimony. The effect of including a mixture on the loading matrix is that each PCT can be modelled by means of a different correlation matrix between diseases. We illustrate the range of the estimates by providing results in Tables 3 and 4 for the posterior mean correlation from the two PCTs, ('A' and ' $\mathrm{B}$ ') which had the greatest difference in the determinant of this estimated correlation matrix.

\begin{tabular}{rrrrrrr}
\hline & Lung (F) & Oral (F) & Lung (M) & Oral (M) & Breast & Cervix \\
\hline Lung (F) & 1.00 & 0.45 & 0.84 & 0.37 & 0.21 & 0.58 \\
Oral (F) & 0.45 & 1.00 & 0.80 & 0.98 & -0.74 & 0.69 \\
Lung (M) & 0.84 & 0.80 & 1.00 & 0.71 & -0.28 & 0.56 \\
Oral (F) & 0.37 & 0.98 & 0.71 & 1.00 & -0.77 & 0.75 \\
Breast & 0.21 & -0.74 & -0.28 & -0.77 & 1.00 & -0.28 \\
Cervix & 0.58 & 0.69 & 0.56 & 0.75 & -0.28 & 1.00 \\
\hline
\end{tabular}

Table 3: Posterior mean estimate of inter-variable correlation for selected PCT 'A' from latent structure mixture spatial model with three latent variables and three mixture groups

\begin{tabular}{rrrrrrr}
\hline & Lung (F) & Oral (F) & Lung (M) & Oral (M) & Breast & Cervix \\
\hline Lung (F) & 1.00 & 0.51 & 0.85 & 0.46 & 0.14 & 0.54 \\
Oral (F) & 0.51 & 1.00 & 0.84 & 0.99 & -0.74 & 0.57 \\
Lung (M) & 0.85 & 0.84 & 1.00 & 0.78 & -0.30 & 0.47 \\
Oral (M) & 0.46 & 0.99 & 0.78 & 1.00 & -0.77 & 0.63 \\
Breast & 0.14 & -0.74 & -0.30 & -0.77 & 1.00 & -0.29 \\
Cervix & 0.54 & 0.57 & 0.47 & 0.63 & -0.29 & 1.00 \\
\hline
\end{tabular}

Table 4: Posterior mean estimate of inter-variable correlation for selected PCT ' $\mathrm{B}$ ' from latent structure mixture spatial model with three latent variables and three mixture groups

As can be seen, the differences are subtle. With the exception of Breast Cancer, these estimated 
correlation matrices are similar to those seen with the MCAR model, the correlation with Lung $(F)$, Oral (F) and Oral (M) are greater in magnitude when estimated by the latent structure model, the Aitchison and Ho (1989) moment estimators are somewhere between either of these estimated. It would also appear that the main differences between these assumed correlation matrices (over which all inter-disease dependence is averaged) are in terms of the relationship between cervical cancer and Oral Cancer ( $M$ and F) as well as Lung Cancer (M).

It is useful in a spatial application to present maps of the geographical distribution of relative risk from the two models. These results are illustrated in Figure 2 for Breast cancer (as well as results from the latent structure mixture model to be explained in section 4.3). Both models achieve a degree of shrinkage in terms of the posterior mean of the relative risks when compared with the raw data. Maps for the other cancer counts reveal a similar story.

\subsection{Latent Structure Mixture Model}

We now present results from our alternative proposed model in which the spatially distributed latent variables are replaced with a mixture distribution (i.e. model (3)). Again we fitted a range of models with differing numbers of latent variables and differing numbers of mixture components. For similar reasons to those discussed in relation to model (4), we chose a three latent variable model. As with model (4), the posterior predictive score tends to favour models with a larger number of mixture components. The posterior predictive loss indicates that, generally speaking, increasing the number of mixture components improves the fit but increases the penalty term. However, Kullback-Leibler tends to favour a two component solution. Figure 3 contains a density plot of the sampled values for the approximate Kullback-Leibler distance between a two component mixture and a one component model and indicates a considerable distance between the two and one component means of the second latent variable.

Given this support from the Kullback-Leibler distance we accept a two component solution despite the fact that this fits slightly less well than higher numbers of components on the Dawid and Sebastiani score, which was estimated as $-8,934.1$ and is comparable to the score from the spatially mixed latent variable model with two mixture groups, but slightly worse than the $-8,923.9$ seen with the three spatial latent variable three mixture group model.

The posterior deviance had mean of 13890.0 , with a 95\% Credible Interval $(13790.0,13890.0)$ which is similar to those seen with all other models. Using the non-proper PPL the fit value $G$ of $58,872.0$ is worse than the value of $55,651.4$ seen in the five component spatial model but is better than the value of $59,854.5$ seen with the three component spatial model. However, the penalty term $P$ is slightly worse than either of them at $24,691.4$ rather than $24,493.1$ or $24,589.2$ respectively.

The model fit diagnostics are therefore very similar to those seen with the mixed latent spatial variables; in both cases the fit can be improved by increasing the number of mixture groups. However, investigation of this model using the Kullback-Leibler distance suggests that these additional groups may be acting in a non-parametric manner.

Summaries of the posterior relative risks from models (3) and (4)for Breast cancer are overlaid in Figure 4. In these terms the two models are roughly similar. However, the models differ substantially when the mixture groupings are interpreted in terms of individual PCTs. For our chosen three component latent structure mixture spatial model posterior mean mixing probabilities for each PCT do not strongly discriminate PCTs as predominantly belonging to any particular component. This is consistent with our earlier observation that the mixture 

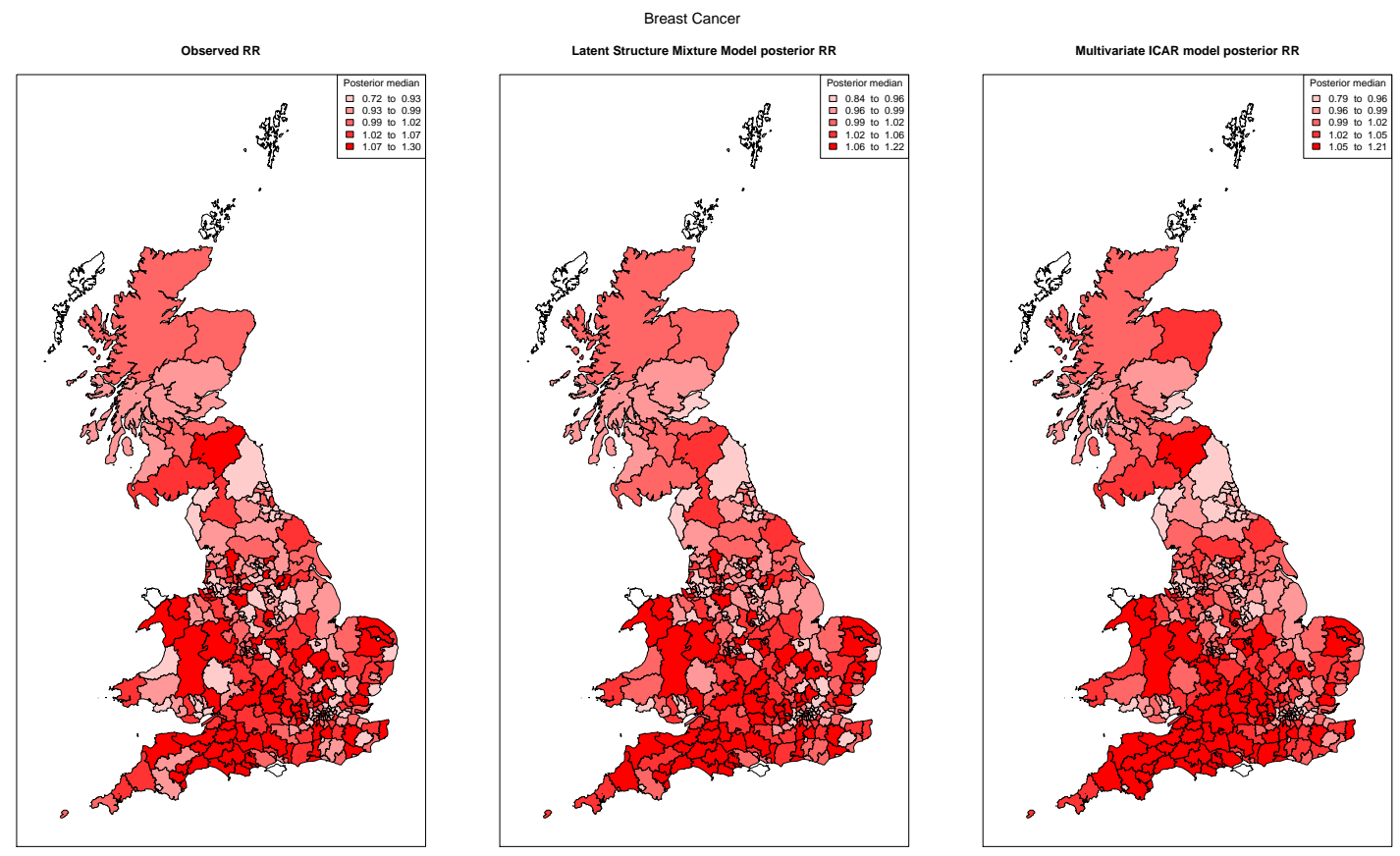

Figure 2: Cervical cancer raw rates and posterior mean relative risk from MCAR model and from latent structure mixture spatial model

\section{Posterior estimates of approximate Kullback-Leibler distance}

Proposal to collapse $\delta_{1}$

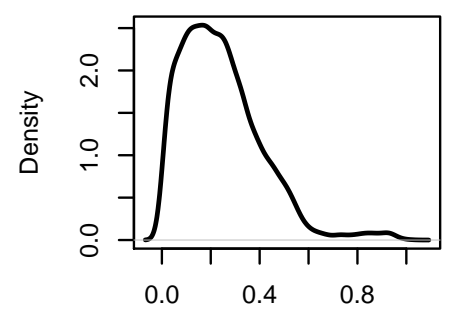

Approx.KL
Proposal to collapse $\delta_{2}$

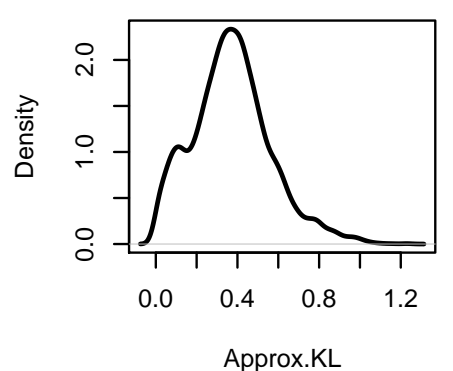

Proposal to collapse $\delta_{3}$

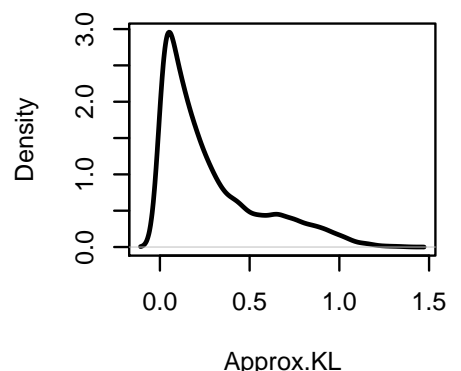

Figure 3: Approximate Kullback Leibler distance between two and one component latent structure mixture model with three latent variables when considering each of the latent variables 
components for this model overlap and do not necessarily have any substantive meaning. This is in strong contrast to what is seen when posterior mean mixing probabilities for each PCT are examined for our chosen two component latent structure mixture model. Figure 5 gives the posterior groupings of PCTs associated with this model. It is clear that although no explicit spatial structure is imposed in this model, the mixture groups appear to be highlighting a spatial patterns that have a substantive interpretation (Scotland and industrial areas in England and Wales). There does therefore appear appear to be some interesting possibilities in using this type of model formulation.

Finally, we present illustrative results demonstrating the out of sample performance of our models. A random 35 PCTs had data removed for a a randomly selected cancer site (Female Lung Cancer). Results are depicted in figure 6 which contrasts the posterior predictive density for the omitted data with the actual data that had been excluded from the model.

In addition to the visual display seen in figure 6 it is most interesting to note that the Dawid and Sebastiani score given in formula (5) for these out of sample predictions was -147.8 for the latent structure spatial model, and -147.7 for the latent structure mixture model. These are strikingly similar values, and it is also noteworthy that both are slightly better than the score from the MVCAR model with additional random effects which had a score of -153.6

\section{Discussion}

We believe that the latent structure mixture models developed in this paper provide a tractable approach to handling situations in joint disease modelling where it may be anticipated that a single dependence structure, either between diseases or across geographical units, is overly restrictive. We also believe that such situations are not uncommon and that aspects of the illustrative cancer morbidity data we have examined substantiate the argument for employing mixture models as a way of avoiding unreasonable exchangeability assumptions. With these data, it is notable that the latent structure mixture models report considerably better model fit diagnostics (in terms of the proper scoring rule used) than an MCAR model even with the addition of an a non-spatially correlated random effect.

More importantly, these models demonstrate slightly better performance in terms of out-ofsample predictions for a randomly selected sub-sample deleted from one of the cancer sites. This confirms the potential for these multivariate models in terms of borrowing strength across variables.

Our models have some analogies to recent work of Hall and Wang (2005) who superimposed a two-component mixture on the latent variable of a univariate GLMM; but we have extended that to multivariate settings where flexibility in modelling a dependence structure is required. Our approach effectively generalises the 'mixture of factor analysers' model (Ghahramani and Hinton, 1996) to one that can deal with non-Gaussian responses. In that sense the models we have developed can be considered as extensions to the latent structure models that are seeing increasing use in biometric applications, such as those presented by Dunson (2003); Wang and Wall (2003); Minozzo and Fruttini (2004); Dunson and Herring (2005).

Our primary focus has been on statistical methodology, rather than identifying any substantive epidemiological issues arising from the particular cancer morbidity data we have examined. That said, there could well be interesting epidemiological distinctions between the areas discriminated using our approach as reported in Section 4. It is quite striking that the areas 


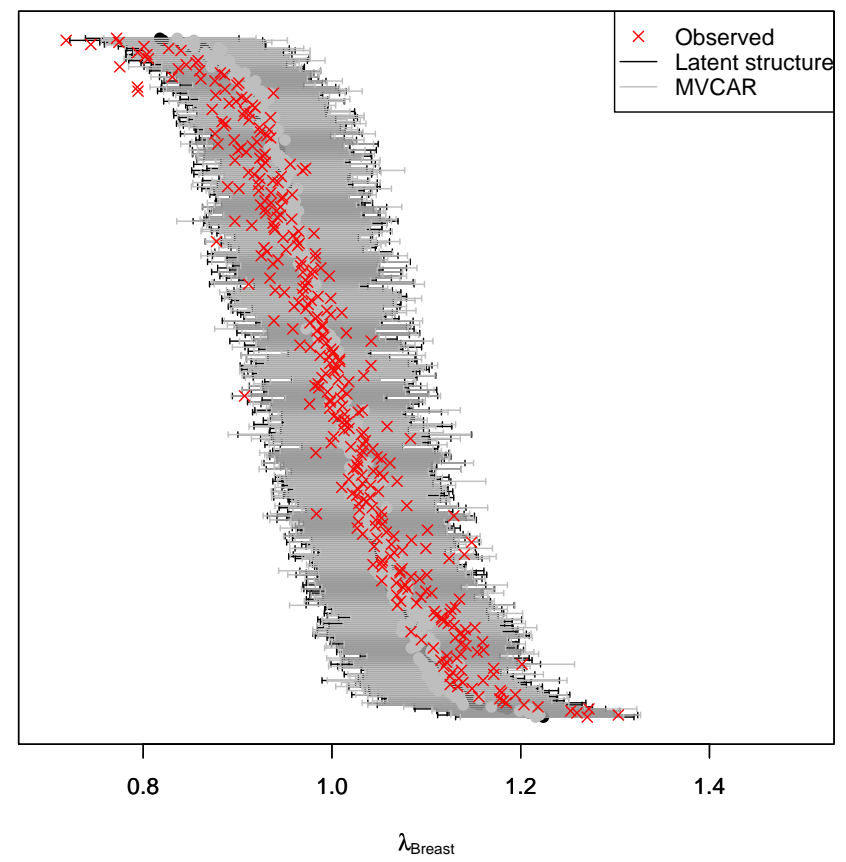

Figure 4: Comparison of posterior relative risk distribution from latent structure mixture spatial model (in black) and latent structure mixture (superimposed in lighter colour)

with lowest probability of group 1 membership tending to correspond to former industrial areas of Scotland, North England and Wales, those PCTs with the highest probability of group 1 membership tending to correspond to more affluent and rural areas in Southern England. This fits well with the epidemiology of these diseases, lifestyle factors such as alcohol and tobacco consumption being more dominant in the non-group 1 areas (hence greater lung and oral cancer) and other factors being responsible for greater breast cancer risk in the group 1 areas. Work in the machine learning literature which approaches 'mixture of factor analysers' by means of 'variational Bayes' algorithms has illustrated the strong potential of models similar to ours in terms of unsupervised classification (Ghahramani and Beal, 2000). However, we are cautious of over-interpretation in this regard; further work is needed in terms of assessing model fit. We judge the two mixture model an appropriate model in terms of Kullback-Leibler distance between mixture components, but it was clear that model fit can be improved (in terms of the Posterior Predictive Score) by increasing the number of mixture components. We suspect that this improved fit may well be attained in a non-parametric manner, and that the "groups" have no substantive interpretation, but clearly much further work is needed in terms of understanding the model fitting process.

We have concentrated on modelling dependency structure and not explicitly addressed use of additional covariate information on geographical units other than routine standardisation for age/sex population structure. We appreciate that in practice it is very likely that relevant additional covariate information will be available on the geographical units concerned. If so, then this can easily be handled by simply including relevant fixed effects into the linear predictor of the models we have proposed and does not present any additional methodological challenges. For example, in the particular illustrative application discussed in this paper one 
Posterior probability for group 1 memberships

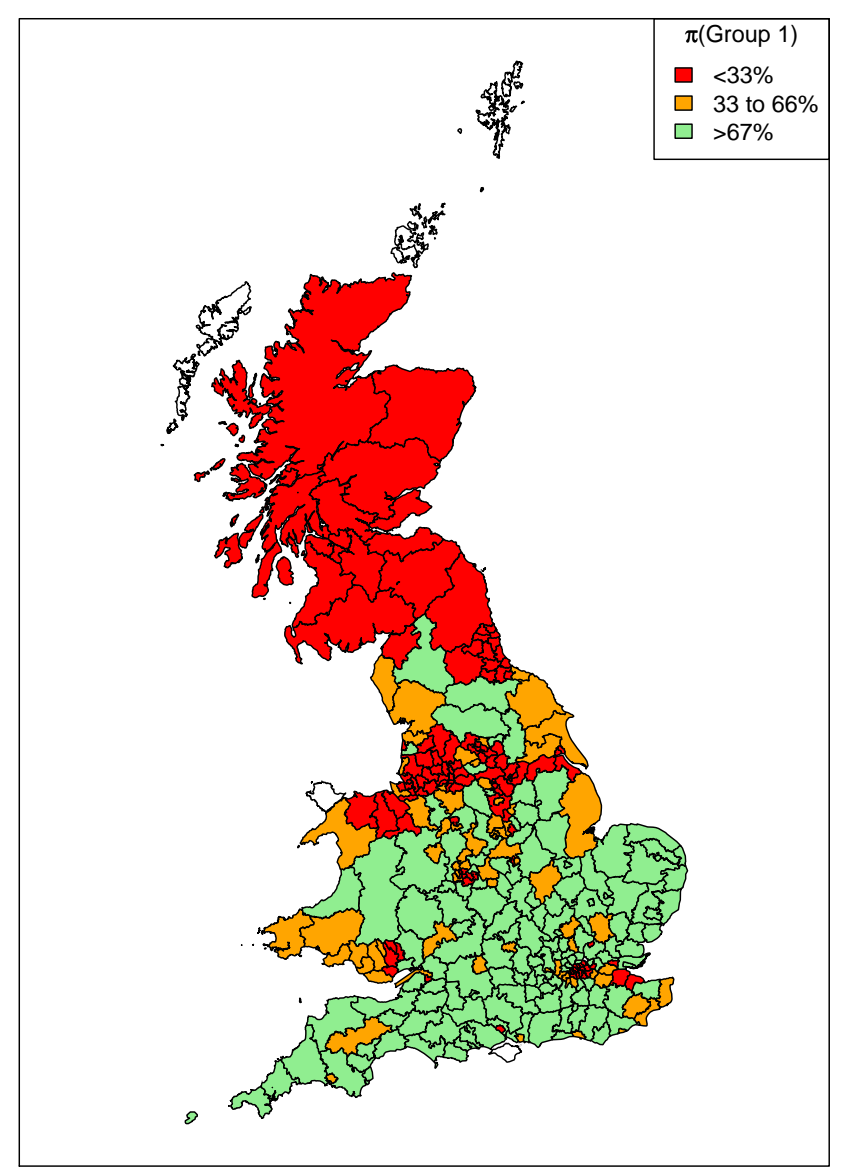

Figure 5: Posterior probability of PCT group membership for latent structure mixture model with two components and three latent variables. 


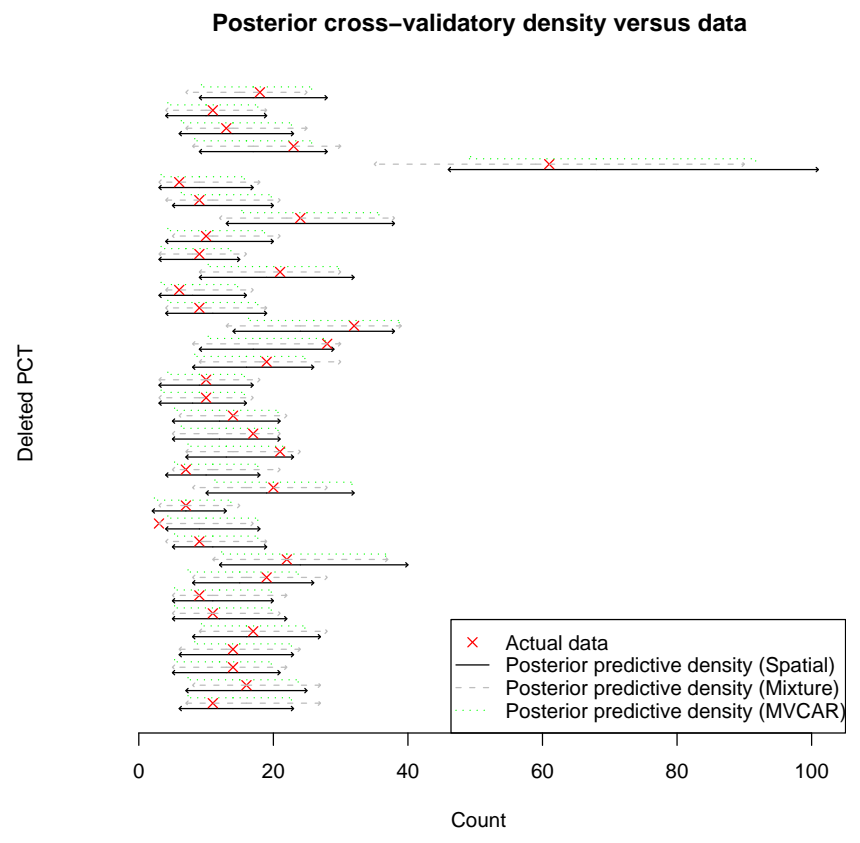

Figure 6: Out of sample posterior predictive density for 35 "PCTs" randomly removed from the Female Lung Cancer site. Predictions given from both the latent structure model containing spatially correlated random effects (solid lines) as well as the latent structure model containing discrete mixtures of random effects (dotted lines). The actual removed data points have been superimposed 
potentially useful modification to the model proposed would have been some accounting for suspected problems with data consistency. Following Best and Wakefield (1999) we could perhaps have incorporated a fixed effect for cancer registry effects. Without that we rely on the questionable assumption that cancer registry effects are common across cancers which may not be reasonable. It is straightforward to incorporate such an allowance for registry effects.

Our models have some similarities to what Viroli (2004) refers to as 'Independent Factor Analysis' models. These have become popular in the signal processing literature where the mixtures are viewed as acting in a non-parametric manner as a way of modelling a nonGaussian latent variable. However, the interaction between the spatial latent variables and mixture latent variables remain an area that requires further exploration. Given that mixtures applied to the loadings may improve model fit in a non-parametric way, it is interesting that a potentially substantive classification can be obtained by examining the latent variable mixtures. Considerable work is needed to deal with these models in such a way that the mixtures on the loadings can be disentangled from the mixtures on the latent variables, but it does appear that the two structures do act differently.

We appreciate that our models could benefit from MCMC algorithmic development. One approach would be to develop custom algorithms based around Structured MCMC (Sargent, 2000). Reversible Jump MCMC is another approach that requires investigation and it should be noted that Sahu and Cheng (2003) have suggested that it may be possible to incorporate considerations about Kullback-Leibler distance into RJMCMC proposal distributions. That said, we doubt that fully automatic RJMCMC methods are feasible to implement with these models, since both the number of mixture components and the number of latent variables are unknown. The potential to consider both mixtures on loadings and on latent variables leads to these choices being confounding in a fully automatic fitting procedure. Our own simulation studies reveal a degree of indeterminacy between the number of latent variables and the number of mixture components. However, it is not clear that RJMCMC approaches are crucial in determining the required number of latent variables (Dunson, 2006). We are also currently considering the importance of the identifiability constraints on the loadings which we have adopted in our models. The logic of parameter expansion suggests that, for predictive models, the identifiability of loadings and latent variables is not an issue, it is their product that has to be identifiable. However, it is important that information to identify the mixture components is preserved and as yet and we have no theoretical results to guide this decision.

In summary, we believe that incorporating a mixture distribution into a latent structure model has considerable potential in modelling multivariate disease rates. The advantages of using a latent structure model relate to the transparent way in which correlation structure is represented in the model allowing the modeller to tune this accordingly. It is less obvious how to do this within, say, the MCAR formulation where the latent structure is not explicit. In this paper we have considered both spatial and non-spatial variations within the latent structure mixture, but other variations could clearly be formulated within the same framework. In terms of the application considered, use of the latent variable mixture approach identified two possible groups of areas which may have substantive epidemiological interpretation. In comparison with MCAR approaches, our latent structure mixture models exhibit broader relative risk posterior distributions, but with means that more closely reflect the observed data. 


\section{Acknowledgements}

We would like to thank personnel in each of the nine cancer registries in England and well as the cancer registries in Scotland and Wales for supplying the data used in this study. We would like to thank the helpful input of the referees, in particular for bringing the work on proper scoring rules (the Dawid and Sebastiani score) to our attention.

\section{References}

Aguilar, O. and M. West (2000). Bayesian Dynamic Factor Models and Portfolio Allocation. Journal of Business and Economic Statistics 18, 338-357.

Aitchison, J. and C. H. Ho (1989). The Multivariate Poisson-Log Normal Distribution. Biometrika 76, $643-653$.

Aitkin, M. and I. Aitkin (2005). Bayesian Inference for Factor Scores. In A. Maydeu-Olivares and J. J. McArdle (Eds.), Contemporary Advances in Psychometrics. New York: Erlbaum.

Banerjee, S., B. P. Carlin, and A. E. Gelfand (2004). Hierarchical Modeling and Analysis for Spatial Data. Boca Raton: Chapman and Hall / CRC.

Beal, M. J. and Z. Ghahramani (2003). The Variational Bayesian EM Algorithm for Incomplete Data: with Application to Scoring Graphical Model Structures. In J. Bernardo, M. Bayarri, J. Berger, A. Dawid, D. Heckerman, A. Smith, and M. West (Eds.), Bayesian Statistics 7. Oxford University Press.

Best, N. and J. Wakefield (1999). Accounting for Inaccuracies in Population Counts and Case Registration in Cancer Mapping Studies. J. R. Statist. Soc. A 162, 363-382.

Celeux, G., M. Hurn, and C. P. Robert (2000). Computational and Inferential Difficulties with Mixture Posterior distributions. J. Am. Statist. Ass. 95, 957-970.

Christensen, W. F. and Y. Amemiya (2002). Latent Variable Analysis of Multivariate Spatial Data. J. Am. Statist. Ass. 97, $302-317$.

Christensen, W. F. and Y. Amemiya (2003). Modeling and Prediction for Multivariate Spatial Factor Analysis. Journal of Statistical Planning and Inference 115, 543 - 564.

Clayton, D. G. and J. Kaldor (1987). Empirical Bayes Estimates of Age-standardised Relative Risks for use in Disease Mapping. Biometrics 43, 671-681.

Czado, C., T. Gneiting and L. Held (2007) Predictive Model Assessment for Count Data. University of Washington Department of Statistics Technical Report Number 518

Dabney, A. and J. Wakefield (2005). Issues in the Mapping of Two Diseases. Statistical Methods in Medical Research 14, 83-112.

Daniels, M. J. and R. E. Kass (2001). Shrinkage Estimators for Covariance Matrices. Biometrics 57, $1173-1184$.

Dawid, A.P. and P. Sebastiani (1999). Coherent Dispersion Criteria for Optimal Experimental Design Annals of Statistics $27,66-81$

Dreassi, E. (2007). Polytomous Disease Mapping to Detect Uncommon Risk Factors for Related Diseases. Biometrical Jnl. 49, 520-529.

Dunson, D. (2003). Dynamic Latent Trait Models for Multidimensional Longitidinal Data. J. Am. Statist. Ass. 98, 555-563. 
Dunson, D. (2006). Efficient Bayesian Model Averaging in Factor Analysis. Technical report, National Institute of Environmental Health Sciences, NC, USA.

Dunson, D. and A. Herring (2005). Bayesian Latent Variables for Mixed Discrete Outcomes. Biostatistics $6,11-25$.

Eberly, L. and B. Carlin (2000). Identifiability and Convergence Issues for Markov chain Monte Carlo Fitting of Spatial Models. Statistics in Medicine 19, 2279-2294.

Gelfand, A. and P. Vounatsou (2003). Proper Multivariate Conditional Autoregressive Models for Spatial Data Analysis. Biostatistics 4, 11-15.

Gelfand, A. E. and S. K. Ghosh (1998). Model Choice: A Minimum Posterior Predictive Loss Approach. Biometrika 85(1), 1-11.

Gelman, A. and D. Rubin (1992). Inference from Iterative Simulation using Multiple Sequences. Statistical Science 7, 457-511.

Geweke, J. (1992). Evaluating the Accuracy of Sampling-Based Approaches to Calculating Posterior Moments. In J. Bernado, J. Berger, A. Dawid, and A. Smith (Eds.), Bayesian Statistics 4. Oxford: Clarendon Press.

Ghahramani, Z. and M. Beal (2000). Variational Inference for Bayesian Mixtures of Factor Analysers. MIT Press.

Ghahramani, Z. and G. E. Hinton (1996). The EM Algorithm for Mixtures of Factor Analysers. Technical Report CRG-TR-96-1, Department of Computer Science, University of Toronto.

Gneiting, T. and A. E. Raftery (2007). Strictly Proper Scoring Rules, Prediction and Estimation. J.Am. Statist. Ass. 102, 359-378.

Green, P. J. (2003). Trans-dimensional Markov chain Monte Carlo. In P. Green, N. L. Hjort, and S. Richardson (Eds.), Highly Structured Stochastic Systems, Number 27 in Oxford Statistical Science Series, pp. 179-198. Oxford: Oxford University Press.

Grzebyk, M., P. Wild, and D. Chouaniere (2004). On Identification of Multi-Factor Models with Correlated Residuals. Biometrika 91(1), 141-151.

Hall, D. B. and L. Wang (2005). Two-Component Mixtures of Generalised Linear Mixed Effects Models for Cluster Correlated Data. Statistical Modelling 5, 21-37.

Hardy, G., J. Littlewood, and G. Plya (1988). Some Theorems Concerning Monotonic Functions (2 ed.)., pp. 83-84. Cambridge, UK: Cambridge University Press.

Heidelberger, P. and P. Welch (1983). Simulation Run Length Control in the Presence of an Initial Transient. Opns Res 31, 1109-44.

Held, L., I. Natario, S. Fenton, H. Rue, and N. Becker (2005). Towards Joint Disease Mapping. Statistical Methods in Medical Research 14, 61-82.

Hogan, J. W. and R. Tchernis (2004). Bayesian Factor Analysis for Spatially Correlated Data, With Application to Summarizing Area-Level Material Deprivation from Census Data. J. Am. Statist. Ass. 99(466), 314-324.

Knorr-Held, L. and N. Best (2001). A Shared Component Model for Joint and Selective Clustering of Two Diseases. J.R. Statist.Soc. A 164, 73-86.

Lee, S.-Y. and X.-Y. Song (2003). Bayesian Model Selection for Mixtures of Structural Equation Models with an Unknown Number of Components. British Journal of Mathematical and Statistical Psychology 56, 145-165. 
Leonard, T. and J. S. Hsu (1992). Bayesian Inference for a Covariance Matrix. The Annals of Statistics 20(4), 1669-1696.

Liu, X., M. M. Wall, and J. S. Hodges (2005). Generalized Spatial Structural Equation Models. Biostatistics 6(4), 539-557.

Lopes, H. and M. West (2004). Bayesian Model Assessment in Factor Analysis. Statistica Sinica 4, 41-67.

McLachlan, G. J. and D. Peel (2000). Finite Mixture Models. New York: J. Wiley and Sons.

Minozzo, M. and D. Fruttini (2004). Loglinear Spatial Factor Analysis: an Application to Diabetes Mellitus Complications. Environmetrics 15, 423-434.

Normand, S.-L. T., M. E. Glickman, and C. A. Gatsonis (1997). Statistical Methods for Profiling Providers of Medical Care: Issues and Applications. J. Am. Statist. Ass. 92, 803-814.

Ogasawara, H. (1996). A Proposal of Poisson Factor Analysis. International Journal of Psychology 31, 48490.

Sahu, S. and R. Cheng (2003). A Fast Distance Based Approach for Determining the Number of Components in Mixtures. The Canadian Journal of Statistics 31, 3-22.

Sargent, D.J., J.S. Hodges and B.P. Carlin (2006). Structured Markov Chain Monte Carlo, Journal of Computational and Graphical Statistics 9, 217-234.

Song, J., M. Ghosh, S. Miaou, and B. Mallick (2006). Bayesian Multivariate Spatial Models for Roadway Traffic Crash Mapping. Journal of Multivariate Analysis 97, 246-273.

Spiegelhalter, D., A. Thomas, and N. Best (1998). WinBUGS version 1.1.1 User Manual. Technical report, MRC Biostatistics Unit, Cambridge.

Viroli, C. (2004). Choosing the Number of Factors in Independence Factor Analysis Model. Metodoloski zvezki 1, 407-418.

Wang, F. and M. M. Wall (2003). Generalized Common Spatial Factor Model. Biostatistics 4, 569-582.

Wedel, M., U. Bockenholt, and W. A. Kamakura (2003). Factor Models for Multivariate Count Data. Journal of Multivariate Analysis 87, 356-369.

Yang, R. and J. O. Berger (1994). Estimation of a Covariance Matrix Using the Reference Prior. The Annals of Statistics 22(3), 1195-1211. 